\title{
ARTICLE
}

\section{Measurement of activation cross sections of aluminum for protons with energies between 0.4 GeV and 3.0 GeV at J-PARC}

\author{
Hiroki Matsuda, Shin-ichiro Meigo and Hiroki Iwamoto \\ JAEA/J-PARC, Shirakata 2-4, Tokai-mura, Naga-gun, Ibaraki, 319-1195, Japan
}

\begin{abstract}
We measured activation cross sections of aluminum for $0.4,1.3,2.2$, and $3.0 \mathrm{GeV}$ protons at J-PARC to obtain basic data for nuclear design of beam windows as the test experiment. It was found that data with higher accuracy than current ones will be measured by using precise beam controls and highly accurate beam monitoring. We compared the experimental results, the evaluated data (JENDL-HE/2007), and calculation results with several intra-nuclear cascade models in PHITS. Although the experimental data agreed with JENDL-HE/2007, the calculations underestimated by about $40 \%$, which could come from the evaporation model (GEM) included in PHITS code. It was found that the calculations with original GEM code show good agreement with the experimental data.
\end{abstract}

Keywords: J-PARC; proton beam transport; activation cross section; aluminum; PHITS; GEM

\section{Introduction}

At the Materials and Life Science Experimental Facility (MLF) of the Japan Proton Accelerator Research Complex (J-PARC) [1] the Japan Spallation Neutron Source [2], and the Muon Science facility (MUSE) [3] have been installed as a MW-class pulsed neutron source. Since 2008, a high-power proton beam of $300 \mathrm{~kW}$ has been delivered. In 2015, we successfully ramped up beam power to $500 \mathrm{~kW}$ and delivered the 1-MW beam to the targets. Neutrons are produced by a 3 $\mathrm{GeV}$ proton beam incident on a mercury target, and muons are obtained by the $3 \mathrm{GeV}$ proton beam hitting a 2 -cm thick carbon graphite target. To efficiently use the proton beam for particle production, both targets are aligned in a cascade scheme, with the graphite target placed $33 \mathrm{~m}$ upstream of the neutron target. For both sources, the $3 \mathrm{GeV}$ proton beam is delivered from a rapid cycling synchrotron (RCS) to the targets by the 3NBT ( $3 \mathrm{GeV}$ RCS to Neutron Facility Beam Transport). Before injection into the RCS, the proton beam is accelerated up to $0.4 \mathrm{GeV}$ by a linear accelerator (LINAC). The beam is accumulated in two short bunches and accelerated up to $3 \mathrm{GeV}$ in the RCS. The extracted $3 \mathrm{GeV}$ proton beam, with a 150-ns bunch width and a spacing of $600 \mathrm{~ns}$, is transported to the muon production target and the spallation neutron source.

In J-PARC, the Transmutation Experimental Facility (TEF), is planned for the development of the target for Accelerator Driven System (ADS) as well. Also, similar

*Corresponding author. Email: matsuda.hiroki@jaea.go.jp proton beam window will be applied at the TEF. For the nuclear design of spallation neutron sources and a transmutation system, decommissioning of the accelerator and TEF facilities, activation cross section data with high accuracy are mandatory for various materials. However, the past experimental data are very few and insufficient in quality. In this paper, we will describe the experiment and results of the activation cross section of aluminium that was irradiated with $0.4,1.3,2.2$, and 3.0 $\mathrm{GeV}$ protons.

\section{Experiment}

\subsection{Setup}

To obtain activation cross section, the experiment was carried at the beam transport from the RCS to the MLF. Sample foils were placed at the beam dump line for the tuning of the RCS. To estimate background radiation, some of the foils were located on the outside of the stage. A linear stage guide was utilized to control the irradiation status. Thin square aluminium foils, $0.1 \mathrm{~mm}$ thick and $25 \mathrm{~mm}$ long, were used as an irradiation target. Each of them was sandwiched by rectangular aluminium foils having a size of $38 \mathrm{~mm} \times 60 \mathrm{~mm}$ and the same thickness as the target. In Figure 1, the actual sample set is shown. Four sets were placed at the entrance of the beam dump, which was made of iron and placed around $12 \mathrm{~m}$ downstream of the foil. As a user facility for supplying secondary beam such as neutrons, $3 \mathrm{GeV}$ beam was delivered to the MLF. For beam tuning of the accelerator and the beam transport, the beam dump was utilized. For the measurement of the cross section, it was 
important to control the beam irradiation condition because the dump was utilized for beam tuning of the accelerator. To control the irradiation condition, the foil was placed on the movable stage in the vacuum chamber. After irradiation, the sample holder was extracted from vacuum chamber then the sample was retracted from the holder. When the vacuum chamber was opened for extraction and placing of foils, a slow leak valve was used to avoid rupture and deformation of the sample.

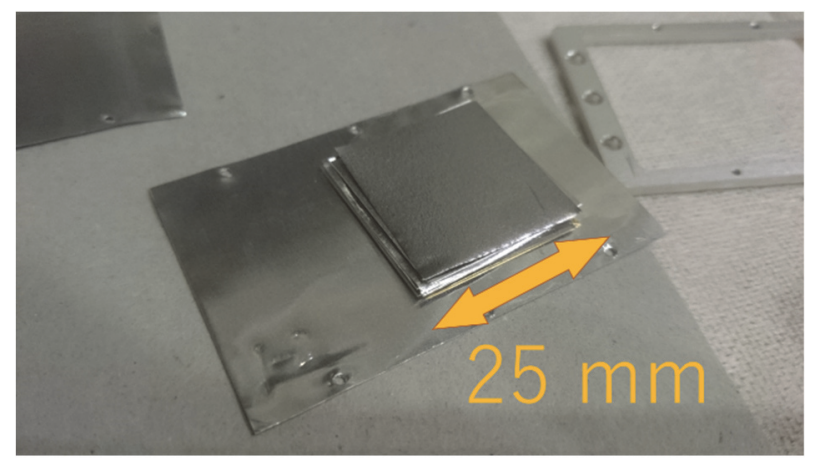

Figure 1. Photo of irradiation sample. Each sample was sandwiched by aluminium foils. The larger aluminium foil covers and fixes the whole sandwiched sample. In total 20 foils ( 5 foils per one sample) were used.

\subsection{Irradiation}

The experiment was carried out at the beam dump placed at the exsit of the RCS in J-PARC. Each foil was irradiated by $0.4,1.3,2.2$, and $3.0 \mathrm{GeV}$ protons. The 0.4 $\mathrm{GeV}$ beam was injected from the LINAC to the RCS. For the tuning of the beam injection into the RCS, the beam can be delivered to the beam dump placed at the exit. The $3 \mathrm{GeV}$ proton was extracted from the RCS. The $1.3 \mathrm{GeV}$, and $2.2 \mathrm{GeV}$ protons were delivered from the RCS acceleration by changing the extraction timing of kicker magnet. The beam width was measured with the multi-wire profile monitor (MWPM). Along the beam transport line, three sets of movable MWPMs are placed to measure the beam profile to the beam dump. The MWPM frame has 31 wires of silicon carbide ( $\mathrm{SiC}$ ) with the spacing pitch of $6 \mathrm{~mm}$ for each horizontal and vertical direction. We employed the $\mathrm{SiC}$ wire having a diameter of $0.1 \mathrm{~mm}$, which had a tungsten core of 0.01 $\mathrm{mm}$ and was coated with $1 \mu \mathrm{m}$ thick pyrolytic carbon film. The wire frame made of aluminium oxide with purity higher than $95 \%$ was selected due to the high radiation resistance. The frame of wires was placed in the vacuum chamber made of titanium, which was selected for its good vacuum characteristics and low activation. To avoid unnecessary irradiation of the wires, the frame could be retracted from the beam and move like the pendulum motion.

By the measurement of the beam width by the MWPMs, the emittance and the Twiss parameter of the beam emittance were acquired. It was shown that the beam width at the foil was $2.5 \mathrm{~mm}$ to $6.5 \mathrm{~mm}$. It should note that the beam position was very stable since the RCS and the beam transport must have quite enough stability to avoid beam loss to deliver the beam from the spallation neutron source.

Repetition of shots was set to $0.4 \mathrm{~Hz}$ to avoid melting of samples on the basis of estimation with a two-dimensional heat transport equation.

\subsection{Analysis}

Decay gamma rays from irradiated samples were measured by the high pure germanium detector (HPGe) immediately after irradiation to observe decay of short-lived nuclei. The main activation products in aluminum were ${ }^{7} \mathrm{Be}$ (half life: $53.22 \mathrm{~d}$ ), ${ }^{22} \mathrm{Na}$ (half life: $2.6018 \mathrm{y}$ ), and ${ }^{24} \mathrm{Na}$ (half life: $14.997 \mathrm{~h}$ ).

The samples were mounted on the acrylic spacer apart from the head of HPGe by about $10 \mathrm{~cm}$ to keep detector-to-sample geometry rigidly. Radioactivity for each product was deduced using the following equation:

$$
A=\exp \left(\lambda t_{i}\right) \frac{\lambda N}{\left(1-\exp \left(-\lambda t_{m}\right)\right) \epsilon I}
$$

where $t_{i}$ was the time interval between the measurement start and the irradiation start time, $N$ was count of $\gamma$-rays peak, $\lambda$ was decay constant, $t_{m}$ was measurement time, $\epsilon$ was detection efficiency, and $I$ was absolute $\gamma$-rays intensity, respectively. Using Eq.1, the activation cross section of specified nuclei was then written as

$$
\sigma=\frac{A}{\lambda N_{p} n}
$$

where $N_{p}$ was the number of protons, and $n$ was the number density of the sample. The detection efficiency was estimated by using ${ }^{152} \mathrm{Eu}$ standard source. Its radioactivity was calculated based on the data in the specification document. Decay data were taken from the latest database managed by IAEA Nuclear Data Services [4]. Irradiation time was about one minute. Beam fluctuation was estimated as less than $1 \%$.

The obtained cross sections were shown in Table 1. Statistical and systematic uncertainties were considered. The list of systematic uncertainties were summarised in Table 2.

Table 1. The present experimental result of ${ }^{7} \mathrm{Be},{ }^{22} \mathrm{Na}$, and ${ }^{24} \mathrm{Na}$ cross sections of aluminum.

\begin{tabular}{cccc}
\hline Proton & \multicolumn{3}{c}{ Cross sections mb } \\
\cline { 2 - 4 } $\begin{array}{c}\text { energy } \\
\mathrm{GeV}\end{array}$ & $\mathrm{Al}(\mathrm{p}, \mathrm{X})^{7} \mathrm{Be}$ & $\mathrm{Al}(\mathrm{p}, \mathrm{X})^{22} \mathrm{Na}$ & $\mathrm{Al}(\mathrm{p}, \mathrm{X})^{24} \mathrm{Na}$ \\
\hline 0.4 & $3.55 \pm 0.45$ & $16.2 \pm 1.0$ & $11.5 \pm 0.8$ \\
1.3 & $7.10 \pm 0.66$ & $11.9 \pm 0.9$ & $10.4 \pm 0.8$ \\
2.2 & $8.77 \pm 0.73$ & $11.7 \pm 0.8$ & $9.8 \pm 0.77$ \\
3.0 & $8.76 \pm 0.73$ & $11.0 \pm 0.8$ & $10.4 \pm 0.8$ \\
\hline
\end{tabular}




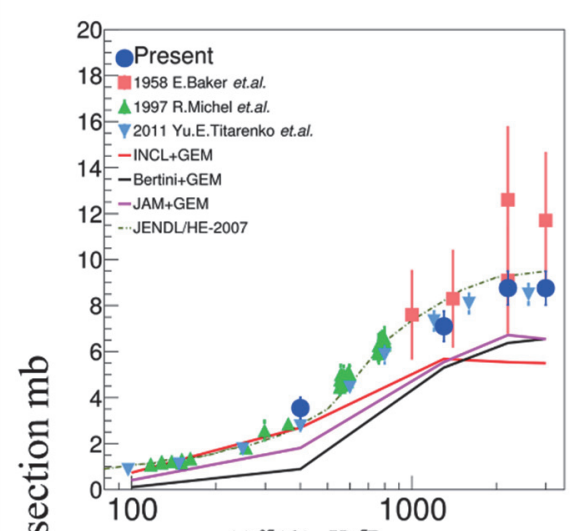

(a) ${ }^{27} \mathrm{Al}(\mathrm{p}, \mathrm{X}){ }^{7} \mathrm{Be}$

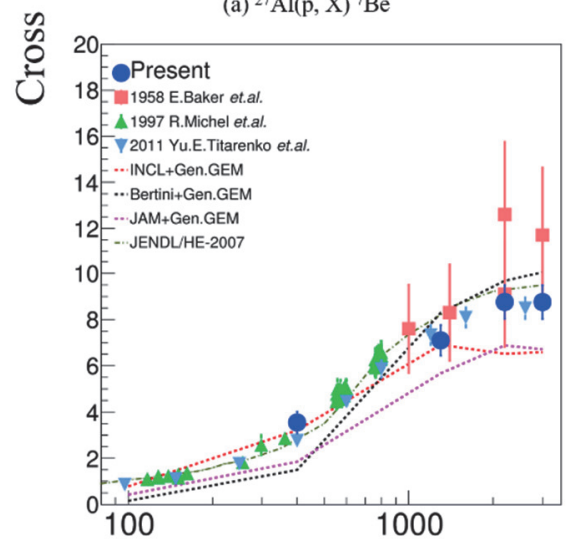

(b) ${ }^{27} \mathrm{Al}(\mathrm{p}, \mathrm{X}){ }^{7} \mathrm{Be}$

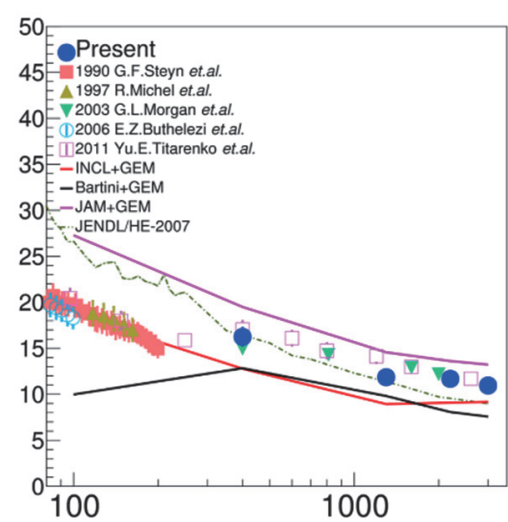

(c) ${ }^{27} \mathrm{Al}(\mathrm{p}, \mathrm{X}){ }^{22} \mathrm{Na}$

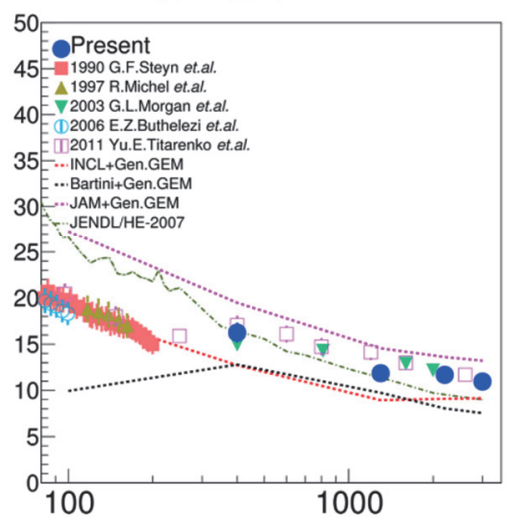

(d) ${ }^{27} \mathrm{Al}(\mathrm{p}, \mathrm{X}){ }^{22} \mathrm{Na}$

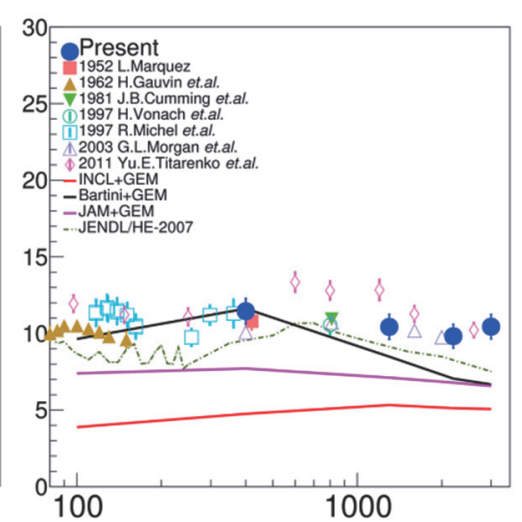

(e) ${ }^{27} \mathrm{Al}(\mathrm{p}, \mathrm{X}){ }^{24} \mathrm{Na}$

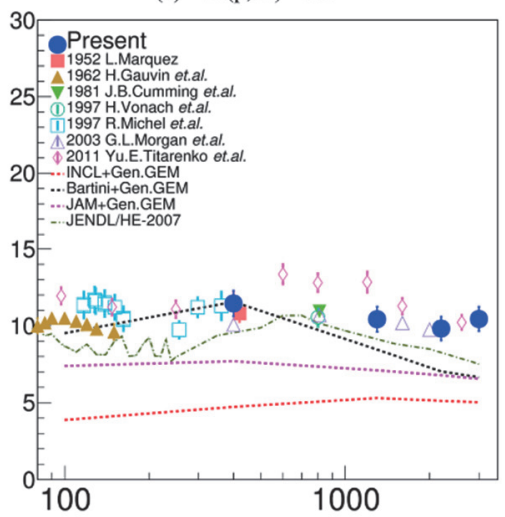

(f) ${ }^{27} \mathrm{Al}(\mathrm{p}, \mathrm{X}){ }^{24} \mathrm{Na}$

Proton energy $\mathrm{MeV}$

Figure 2. Comparison of activation cross sections obtained by the present experiment (full circle) with other experiments [8]. Also, calculations with original PHITS and modified PHITS (denoted as Gen. GEM) are shown in upper and bottom side, respectively.

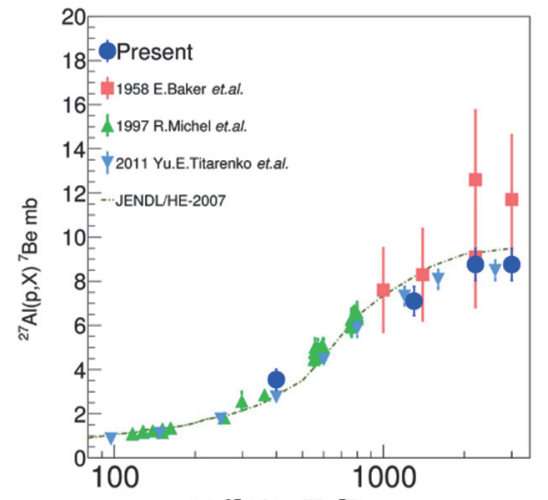

(a) ${ }^{27} \mathrm{Al}(\mathrm{p}, \mathrm{X}){ }^{7} \mathrm{Be}$

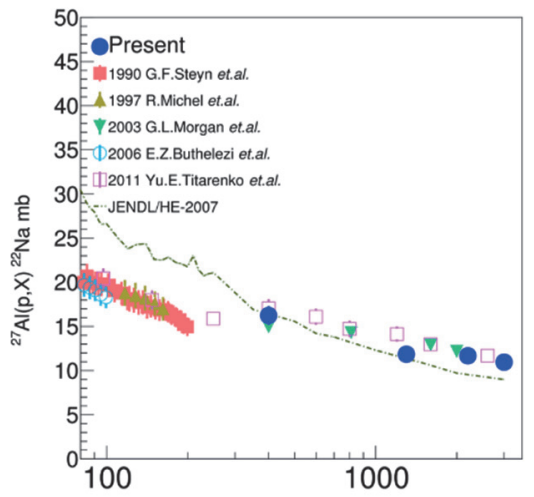

(b) ${ }^{27} \mathrm{Al}(\mathrm{p}, \mathrm{X}){ }^{22 \mathrm{Na}}$

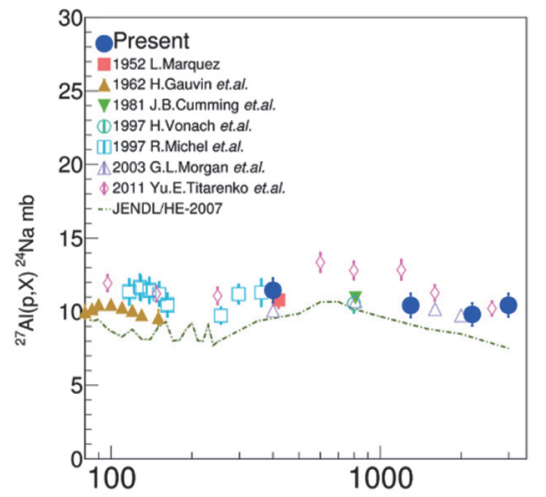

(c) ${ }^{27} \mathrm{Al}(\mathrm{p}, \mathrm{X}){ }^{24} \mathrm{Na}$

Proton energy $\mathrm{MeV}$

Figure 3. Comparison of nuclide activation cross sections of JENDL/HE-2007 [9] with the present and other experiments [8].

PHITS code [5] was employed to calculate the activation cross sections of aluminium with three intranuclear cascade models (Bertini, INCL [6], and JAM). Generalised Evaporation Model (GEM) [7] was used as evaporation model.

\section{Results and discussion}

In Figures 2 and 3, the measured, calculated and evaluated cross sections for ${ }^{27} \mathrm{Al}(\mathrm{p}, \mathrm{X})^{7} \mathrm{Be}$, ${ }^{27} \mathrm{Al}(\mathrm{p}, \mathrm{X}){ }^{22} \mathrm{Na}$, and ${ }^{27} \mathrm{Al}(\mathrm{p}, \mathrm{X}){ }^{24} \mathrm{Na}$ reactions are shown. In the same manner, cross sections of JENDL/HE-2007 are also shown in Figure 3 with the same scale. The 
energy range of JENDL/HE-2007 data is expanded to 3 $\mathrm{GeV}$. Comparing with other experimental data, the present data have smaller uncertainties than others thanks to highly stabilized and controlled proton beam. Their values are also consistent with others.

Table 2. Systematic uncertainties of the present experiment. Decay gamma data mean the data uncertainty of the database.

\begin{tabular}{lc}
\hline & $\begin{array}{c}\text { Relative } \\
\text { uncertainty } \%\end{array}$ \\
\hline Number of protons & 1 \\
Number density of target & 5 \\
Self-absorption of gamma-rays & 1 \\
Decay gamma data & 0.1 \\
Detection efficiency & 3 \\
Beam fluctuation & 1 \\
\hline Total & 6.1 \\
\hline
\end{tabular}

Therefore, the irradiation experiment at the 3NBT beam dump line will be promising and ready for the full-time measurement for various materials.

It was found that the GEM model utilized in PHITS code slightly differs from the original one. Thus, we implemented the original one into PHITS code, then calculated the activation cross sections. They are superposed in Figures 2(b), 2(d), and 2(f) respectively. It is shown that especially for ${ }^{7} \mathrm{Be}$ production the calculated ones are drastically improved by about $40 \%$ (see Figures 2(a) and 2(b)) though the one with INCL still underestimates above $2 \mathrm{GeV}$. On the other hand, the ones for ${ }^{22} \mathrm{Na}$ and ${ }^{24} \mathrm{Na}$ productions are merely affected since sodium isotopes are coming from fragmentation or spallation reactions rather than evaporation one. The current PHITS version adopts statistical multi-fragmentation model (SMM) [10]. SMM could affect the sodium production since sodium is produced by fragmentation process. Future work will focus on comparison with other different models including SMM and quantum molecular dynamics (QMD) [11].

\section{Conclusion}

For the improvement of nuclear design for spallation neutron sources and transmutation systems, nuclear cross section data are of importance. The activation cross sections of aluminium for ${ }^{7} \mathrm{Be},{ }^{22} \mathrm{Na}$, and ${ }^{24} \mathrm{Na}$ production were measured for $0.4,1.3,2.2$, and $3.0 \mathrm{GeV}$ protons to investigate the availability of the 3 NBT beam dump line in J-PARC as an irradiation place. The present experimental results are in good agreement with other experimental ones with a smaller uncertainty than other experiments.

The results of the present experiments were compared with the evaluated nuclear data and the calculation. It was found that JENDL-2007/HE was in good agreement with the present results. On the other hand, it was shown that the calculation with PHITS underestimates the present ones.

However, the production cross section of ${ }^{7} \mathrm{Be}$ calculated with the revised PHITS in which the original GEM was implemented showed good agreement with the present one, especially above $1 \mathrm{GeV}$. For sodium production cross sections, however it was found the revised PHITS does not have significant effect since it is mainly produced by the direct reaction. In this calculation, SMM options were not applied. Not only the difference in this option but also in QMD model should also be considered. We will obtain the cross sections for beryllium, indium, tungsten, and gold which are contained in materials irradiated by the proton beam together with aluminium.

In future works, measurement of the noble gas production (especially $\mathrm{Xe}$ ) reaction for lead and bismuth will be carried out for parameter determination of noble gas treatment for TEF, which will employ a lead-bismuth eutectic target for the development of the ADS.

\section{Acknowledgments}

The authors are grateful to Mr. Nishikawa and the members of Nippon Advanced Technology (NAT) for technical assistance with the experiments for Drs. Saha, Hotchi, and Harada for tuning of the RCS to carry out the present experiment. We also thanks to Drs. Yoshimoto and Kai for the management of activation measurement by HPGe.

\section{References}

[1] The Joint Project Team of JAERI and KEK, JAERI-Tech. 99-56, (1999).

[2] Y. Ikeda, Nucl. Instrum. Meth. A 600 (2009), p.1.

[3] Y. Miyake, et al., Physica. B 404 (2009), p.957.

[4] IAEA NDS, Chart of Nuclides, www-nds.iaea.org/livechart/, 10. July. 2017 visited.

[5] T. Sato, et al., J. Nucl. Sci. Technol. 50(9) (2013), pp.913-923.

[6] A. Boudard, et al., Phys. Rev. C 80 (2013), 014606.

[7] S. Furihata, Nucl. Instrum. Meth. B 171 (2000) p.251.

[8] IAEA, Experimental nuclear reaction data, www-nds.iaea.org/exfor/exfor.html, Accession Numbers: C0255003, O0276009, A0902007, July/10/2017 visited.

[9] Y. Watanabe et al., Journal of the Korean Physical Society 59(2) (2011), pp.1040-1045.

[10]T. Ogawa et al., Nuclear Instruments and Methods in Physics Research A 723 (2013), pp.36-46.

[11]K. Niita et al., Physics Review C 52 (1995), p.2620. 